\title{
Non-Peptide Arginine-Vasopressin Antagonists (Vaptans) for the Treatment of Hyponatremia in Neurocritical Care: A New Alternative?
}

\author{
Jeremy D. Fields $\cdot$ Anish Bhardwaj
}

Published online: 8 May 2009

(C) Humana Press Inc. 2009

The nonapeptide hormone arginine-vasopressin (AVP), also known as antidiuretic hormone $(\mathrm{ADH})$, is the primary regulator of body water content and plasma osmolality. It is synthesized within two hypothalamic nuclei (supraoptic and paraventricular) and stored in the neurohypophysis. Small (parvocellular) vasopressin-containing neurons in the anterior hypothalamus give rise to a complex fiber system that extends throughout the brain. Utilizing immunohistochemical and audioradiographic techniques, extra-hypophyseal vasopressinergic pathways in the brain have been demonstrated that may allow for independent release of central as well as systemic AVP. It has been postulated that these pathways influence water regulation and water permeability in nonneuronal brain cells (glia) $[1,2]$.

Effects of AVP are mediated through three receptor subtypes, each of which belongs to the rhodopsin-like classA G-protein-coupled receptor family [3]. The V1a receptors are primarily located within the vascular smooth muscle and their stimulation results in vasoconstriction. They are also expressed within the brain where they may play an important role in brain water homeostasis and the evolution of brain edema [4], as well as in the myocardium, platelets,

J. D. Fields · A. Bhardwaj

Neurosciences Critical Care Program, Department of Neurology, Oregon Health \& Science University, Portland, OR, USA

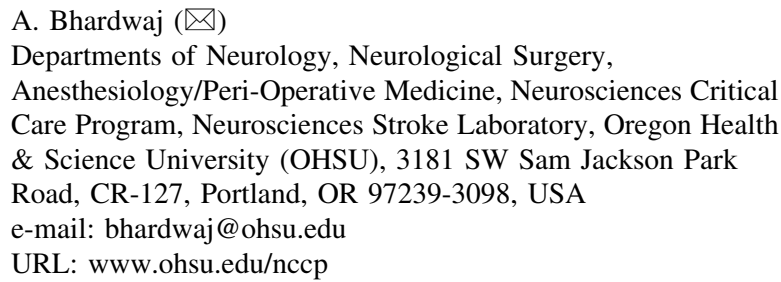

hepatocytes, superior cervical ganglion, the uterus, and cells of the adrenal glands [3,5]. The V1b (formerly designated V3) receptors are found within the anterior pituitary, adrenal medulla, brain, and pancreas and are implicated in the neurohumoral regulation of centrally and peripherally mediated stress response $[3,5]$. V2 receptors are expressed predominantly in basolateral membrane of the renal collecting tubule and to a lesser degree within type 2 pneumocytes and the vascular endothelium [3, 5]. AVP regulates water homeostasis through its interaction with the $\mathrm{V} 2$ receptors in the renal collecting tubule $[3,5,6]$. Binding of AVP to the $\mathrm{V} 2$ receptor activates adenylate cyclase, increasing intracellular concentration of cyclic adenosine monophosphate (cAMP), leading to stimulation of protein kinase A (PKA). PKA increases water resorption by both immediate and delayed mechanisms, namely: (1) phosphorylation of the aquaporin-2 (AQP 2) channel by PKA results in immediate translocation of AQP 2 to the cell membrane and (2) downstream effects resulting in increased expression of AQP-2 within hours [6].

The release of AVP from the neurohypophysis is exquisitely sensitive to plasma osmolality. AVP is secreted at low levels until plasma osmolality reaches approximately $280 \mathrm{mOsm} / \mathrm{kg}$. Above this threshold, the secretion of AVP increases approximately linearly to a maximum output at a serum osmolality of about $295 \mathrm{mOsm} / \mathrm{kg}$, resulting in maximum antidiuresis (urine osmolality $>800 \mathrm{mOsm} / \mathrm{kg}$ ) [6]. In addition to plasma osmolality, AVP may be released as a normal physiologic response to decreased blood pressure or effective circulating volume ( $>10 \%$ from baseline), nausea, pain, stress, hypoxia, fear, and anxiety [6, 7]. Finally, inappropriate release of AVP may occur in association with a variety of neoplasms and drugs, as well as with diseases affecting the central nervous system, peripheral nervous system, or lungs [7]. 
Because of its fundamental role in increasing free water resorption by the kidney (thereby decreasing plasma osmolality), antagonism of the $\mathrm{V} 2$ receptor is a rational target for drugs to combat hyponatremia. Distinct from the currently available diuretics, which result in elimination of both free water and electrolytes ("natriuresis"), compounds acting at the $\mathrm{V} 2$ receptor cause elimination of free water only ("aquaresis"). By preserving plasma electrolytes but decreasing total body free water, aquaresis reliably increases plasma osmolality and the concentration of sodium, the major determinant of plasma osmolality [3, 8]. Although vasopressin antagonists have been available since the 1960s, the original compounds were peptides which were limited by poor penetration into the central nervous system and more importantly, by a tendency to exhibit agonist effects when given chronically. Developed in the 1990s, the nonpeptide AVP antagonists-also referred to as the vaptans-overcame these limitations [3]. This class of drugs now includes those that selectively antagonize the V1a receptor (reclovaptan, OPC-21268, SR-49059), the V1b receptor (SSR-149415), the V2 receptor (lixivaptan, satavaptan, and tolvaptan), and both the V1a/V2 receptor (conivaptan, mozavaptan) [3, 6, 8]. Thus far, only conivaptan has been approved for clinical use in the United States. Mozavaptan is available in Japan.

Irrespective of its etiology, hyponatremia in patients with neuro-injury may be deleterious for numerous reasons. Hyponatremia is associated with a poor clinical outcome, although it is not known whether this is causative or a marker for severity of the underlying neurological disease. There is abundant experimental and clinical evidence to support institution and maintenance of a "euvolemic hyperosmolar" state as a therapeutic principle in patients with neuro-injury. Acute decreases in serum sodium concentration can cause headache, nausea, muscle cramps, and mental status change, or in very severe cases, seizure or coma due to cerebral edema. Furthermore, in patients with pre-existing cerebral edema, a fall in serum sodium may worsen the underlying edema, and exacerbate mass effect leading to uncontrolled intracranial hypertension. Within $48 \mathrm{~h}$, as part of a process known as regulatory volume decrease (RVD), the brain compensates for hyponatremia by releasing osmotically active substances into the interstitial space, establishing an osmotic gradient to drive water out of swollen brain cells and into the interstitium. While this adaptation is beneficial during the period of chronic hyponatremia, if rapid correction of serum sodium occurs before RVD is reversed, the potentially catastrophic osmotic demyelination syndromes (central and extrapontine myelinolysis) can occur [6, 10].

In the neurocritical care setting, hyponatremia is most often due to two disease processes-the syndrome of inappropriate $\mathrm{ADH}$ release $(\mathrm{SIADH})$ and cerebral salt wasting (CSW) [9]. SIADH results from excessive release of AVP, culminating in excess resorption of free water and consequently, a dilutional hyponatremia with slight volume expansion. CSW, as opposed to SIADH, is caused by inappropriate loss of sodium by the kidney. Because free water follows sodium, there is a net decrease of both sodium and water with resultant hypovolemia andbecause the loss of sodium exceeds that of free water (urine osmolality exceeds serum osmolality) — the plasma concentration of sodium decreases. The net result is hypovolemic hyponatremia. The underlying cause of CSW is thought to be the release of circulating natriuretic factors such as brain natriuretic peptide, atrial natriuretic peptide, or ouabain-like substance [10].

In recent issues of Neurocritical Care, two groups have reported their preliminary experience with conivaptan in a diverse patient population (ischemic stroke, subarachnoid hemorrhage $(\mathrm{SAH})$, other vascular lesions, brain tumor, traumatic brain injury, infection, hydrocephalus, GuillainBarre Syndrome) in the neurocritical care setting. Although both groups included patients with $\mathrm{SAH}$, each attempted to exclude those with CSW or vasospasm. Murphy et al. [11] describe the effect of 25 doses of conivaptan (20 or $40 \mathrm{mg}$ bolus given $\geq 12 \mathrm{hr}$ apart) in 19 patients with hyponatremia. In this study, $71 \%$ of patients responded to a single bolus of conivaptan with at least a $4 \mathrm{mEq} / 1$ increase in serum sodium (mean $5.8 \pm 3.2 \mathrm{mEq} / \mathrm{l}$ ) at $12 \mathrm{~h}$; a sustained response $>72 \mathrm{~h}$ was seen in $69 \%$ although the clinical effect as evaluated by urine specific gravity peaked at $2 \mathrm{~h}$ and returned to baseline by $12 \mathrm{~h}$. Wright et al. [12] report the effect of conivaptan $(20 \mathrm{mg}$ bolus followed by infusion of 20 or $40 \mathrm{mg} /$ day) in 22 patients with hyponatremia. This treatment regimen with conivaptan induced at least a $6 \mathrm{mEq} / 1$ increase in serum sodium in $86 \%$ of patients. In both investigations, side effects and adverse events were relatively rare. Overly rapid correction of serum sodium was reported in only one of 41 patients described in these reports $(13 \mathrm{mEq} / \mathrm{l}$ in $24 \mathrm{~h})$. Likewise, only one patient developed symptomatic hypovolemia, likely because maintenance fluids were continued in nearly all patients. Infusion site reactions necessitating IV site change occurred in 5/22 patients receiving the protocol with bolus followed by continuous infusion, but were not observed in the bolus only protocol. Neither study reports the number of patients with significant decreases in serum sodium after medication withdrawal; however, Wright et al. indicate that a subset of patients showed sharp declines in serum sodium after the medication was discontinued.

Although these two reports suggest that conivaptan may be useful in certain situations in neurocritical care, several considerations and limitations need attention. First, in both studies, many different neurologic disease processes (both central and peripheral) and types of hyponatremia are 
considered together. Given the small sample size, it is not possible to identify specific diseases or etiologies more or less amenable to treatment with conivaptan. Given the potential difficulty in discriminating SIADH from CSW, caution in administering AVP antagonists to patients with SAH (especially those at risk for vasospasm) is warranted. Second, concurrent administration of other therapies for hyponatremia (hypertonic saline, oral salt tablets, and fludrocortisone) makes it difficult to delineate the relative effect of conivaptan from that of these other treatment modalities and to know how relative dosages should be adjusted if combination therapy is desired. Third, a rapid decrease seen in several patients after withdrawal of therapy could result in rebound cerebral edema.

What will ultimately be the role of conivaptan and other V2 antagonists in neurocritical care? In SIADH and other forms of euvolemic (or, arguably mildly hypervolemic) hyponatremia, these therapeutic agents have demonstrated efficacy; on the other hand, standard therapy (fluid restriction, hypertonic saline or normal saline, with or without furosemide, salt tablets, fludrocortisone) is cheap and effective, and therefore conivaptan may be reserved primarily for refractory cases or for synergy when urgent correction is necessary. When oral vaptans become available, a subset of patients with chronic symptomatic SIADH may benefit from controlled intravenous in-hospital therapy followed by oral therapy as an outpatient. It is in hypervolemic hyponatremia that the rationale for conivaptan therapy is strongest. In this group, pure aquaresis may be the best mode of preserving intravascular volume while eliminating excess total body free water. Several studies in acute heart failure, for instance, have shown improvements in pulmonary capillary wedge pressure and right atrial pressure without decreases in systolic pressure or creatinine elevation [13]. The use of the medication in hypovolemic hyponatremia (as in CSW) is to be avoided because it could exacerbate volume depletion. As a general rule, with the use of these agents it is imperative that close attention be paid to the volume status to maintain a "euvolemic hyperosmolar" state in patients with neuro-injury.

From a mechanistic perspective, in addition to blocking AVP at the V2 receptor, some of the most exciting neurological applications of vaptans may result from modulation of other cellular targets. V1b receptor antagonists have anxiolytic properties and are being evaluated for use in depression and anxiety disorders. Perhaps most intriguingly, the V1a receptor has recently been implicated in the formation of brain edema via the perivascular pool of aquaporin-4 (AQP-4) [14]. In animal models, decreasing the expression of AQP-4 either by inhibition of the V1a receptor or knock-out of the AQP-4 gene itself leads to the reduction of edema and intracranial pressure following neurotrauma [13] and stroke [15, 16]. Experimental studies in animal models of neuro-injury suggest the anti-edema effects following treatment with nonpeptide AVP antagonists are related to inhibition of AVP at the V1a receptor $[17,18]$. Therefore, in addition to their role in the treatment of euvolemic or hypervolemic hyponatremia in neurocritical care, these agents may ultimately find an important niche in the armamentarium of therapies for the treatment of brain edema.

\section{References}

1. Chang Y, Chen TY, Chen $\mathrm{CH}$, et al. Plasma arginine-vasopressin following experimental stroke: efffect of osmotherapy. J Appl Physiol. 2006;100:1445-51. doi:10.1152/japplphysiol.00763.2005.

2. Niermann H, Amiry-Moghaddam M, Holtoff K, et al. A novel role of vasopressin in the brain: modulation of activity-dependent water flux in the neocortex. J Neurosci. 2001;21:3045-51.

3. Decaux G, Soupart A, Vassart G. Non-peptide arginine-vasopressin antagonists: the vaptans. Lancet. 2008;371:1624-32. doi:10.1016/S0140-6736(08)60695-9.

4. Bloch O, Manley GT. The role of aquaporin-4 in cerebral water transport and edema. Neurosurg Focus. 2007;22:E3. doi:10.3171/ foc.2007.22.5.4.

5. Holmes CL, Landry DW, Granton JT. Science review: vasopressin and the cardiovascular system part 1-receptor physiology. Crit Care. 2003;7:427-34. doi:10.1186/cc2337.

6. Bhardwaj A. Neurological impact of vasopressin dysregulation and hyponatremia. Ann Neurol. 2006;59:229-36. doi:10.1002/ ana.20788.

7. Ellison DH, Berl T. Clinical practice. The syndrome of inappropriate antidiuresis. N Engl J Med. 2007;356:2064-72. doi:10.1056/NEJMcp066837.

8. Ali F, Guglin M, Vaitkevicius P, Ghali JK. Therapeutic potential of vasopressin receptor antagonists. Drugs. 2007;67:847-58. doi:10.2165/00003495-200767060-00002.

9. Rabinstein AA, Wijdicks EF. Hyponatremia in critically ill neurological patients. Neurologist. 2003;9:290-300. doi:10.1097/ 01.nrl.0000095258.07720.89.

10. Nathan BR. Cerebral correlates of hyponatremia. Neurocrit Care. 2007;6:72-8. doi:10.1385/NCC:6:1:72.

11. Murphy T, Dhar R, Diringer M. Conivaptan bolus dosing for the correction of hyponatremia in the neurointensive care unit. Neurocrit Care. 2009 Jan 4. [Epub ahead of print].

12. Wright WL, Asbury WH, Gilmore JL, Samuels OB. Conivaptan for hyponatremia in the neurocritical care unit. Neurocrit Care. 2008 Nov 12. [Epub ahead of print].

13. Udelson JE, Orlandi C, Ouyang J, et al. Acute hemodynamic effects of tolvaptan, a vasopressin V2 receptor blocker, in patients with symptomatic heart failure and systolic dysfunction: an international, multicenter, randomized, placebo-controlled trial. J Am Coll Cardiol. 2008;52(19):1540-5. doi:10.1016/j.jacc.2008.08.013.

14. Zeynalov E, Chen $\mathrm{CH}$, Froehner SC, et al. The perivascular pool of aquaporin-4 mediates the effect of osmotherapy in postischemic cerebral edema. Crit Care Med. 2008;36:2634-40. doi: 10.1097/CCM.0b013e3181847853.

15. Trabold R, Krieg S, Scholler K, Plesnila N. Role of vasopressin $\mathrm{v}(1 \mathrm{a})$ and $\mathrm{v}(2)$ receptors for the development of secondary brain damage after traumatic brain injury in mice. J Neurotrauma. 2008;25(12):1459-65.

16. Kleindienst A, Fazzina G, Dunbar JG, Glisson R, Marmarou A. Protective effect of the v1a receptor antagonist sr49059 on brain edema formation following middle cerebral artery occlusion in 
the rat. Acta Neurochir Suppl (Wien). 2006;96:303-6. doi: 10.1007/3-211-30714-1_65.

17. Molnár AH, Varga C, Berkó A, et al. Prevention of hypoxic brain oedema by the administration of vasopressin receptor antagonist OPC-31260. Prog Brain Res. 2008;170:519-25. doi:10.1016/ S0079-6123(08)00439-1.
18. Ikeda Y, Teramoto A, Nakagawa Y, et al. Attenuation of cryogenic induced brain oedema by arginine vasopressin release inhibitor RU51599. Acta Neurochir (Wien). 1997;139(12):11739. doi:10.1007/BF01410979. 